\title{
Cyclone-anticyclone asymmetry of large-scale wakes in the laboratory
}

\author{
G. Perret, A. Stegner, and M. Farge \\ Laboratoire de Météorologie Dynamique, CNRS, ENS, 24 rue Lhomond, 75005 Paris, France \\ T. Pichon \\ UME, ENSTA Centre de l'Yvette, Chemin de la Hunière, 91120 Palaiseau Cedex, France
}

(Received 4 July 2005; accepted 31 January 2006; published online 31 March 2006)

\begin{abstract}
We performed an experimental study of large-scale wakes in a rotating shallow-water layer. Standard particle image velocimetry was used to measure the horizontal velocity field, while a laser-induced fluorescence technique was used to measure the geopotential deviation (i.e., the interface deviation). According to these measurements, we were able to quantify the dynamics in a wide region of parameter space beyond the quasi-geostrophic regime. For obstacles larger than the deformation radius and with small Rossby numbers, a significant asymmetry occurs in the wake between cyclonic and anticyclonic vortices. These parameters correspond to a frontal geostrophic regime with the relative interface deviation being larger than 0.1-0.2. In this case, anticyclones remain coherent and circular, whereas cyclones tend to be elongated and distorted. More surprisingly, for some extreme cases, coherent cyclones do not emerge at all, and only an anticyclonic vortex street appears several diameters behind the obstacle. The transition from a quasi-geostrophic to a frontal geostrophic regime is characterized by a strong increase in the Strouhal number, which can reach a value up to 0.6. Hence, we found that a large-scale wake could differ strongly from the classical Karman street when the relative geopotential deviation becomes larger than the Rossby number. (C) 2006 American Institute of Physics. [DOI: 10.1063/1.2179387]
\end{abstract}

\section{INTRODUCTION}

Several coherent structures encountered in the oceans or in the atmosphere of giant planets have characteristic scales larger than the local deformation radius. The latter, also called the Rossby radius, is an intrinsic scale that controls the dynamics of rotating and stratified flows. The deformation radius depends on the vertical structure of the flows and may vary from a few tens of kilometers in the ocean to a few thousand of kilometers in the Earth's atmosphere or in that of giant planets. Large-scale flows correspond to a wide variety of geophysical flows having their characteristic scale larger than the local deformation radius. In such cases, an asymmetry is often observed in the dynamics and in the morphology of cyclonic and anticyclonic structures. For instance, the famous long-lived eddies formed in the zonal circulation of outer planets such as Jupiter (Great Red Spot, White oval ${ }^{1,2}$ ), Saturn (Great Dark Spot ${ }^{3}$ ), or Uranus ${ }^{4}$ are anticyclones, whereas cyclones are generally smaller and more elongated. In the Earth's ocean, large-scale eddies such as submesoscale coherent vortex, ${ }^{5}$ meddies, and swoddies ${ }^{6}$ are mostly anticyclonic.

In geophysical flows, several mechanisms can induce a dynamical selection between cyclones and anticyclones. For instance, the predominance of anticyclones could be due to a nonlinear $\beta$ effect. ${ }^{7-11}$ However, in the ocean, the influence of $\beta$ on the submesoscale vortices is weak, and some nonlinear mechanism should act on the $f$ plane. Several studies have considered the simplest model that can take into account ageostrophic or large-scale effects; namely, the rotating shallow-water model in the $f$ plane. As far as shallowwater decaying turbulence is concerned, Polvani et al. ${ }^{12}$ and
Arai and Yamagata ${ }^{13}$ have shown that the departure from quasi-geostrophy leads to a significant asymmetry for which anticyclonic vortices are circular and robust, while cyclones are more distorted and elongated. The parameter that governs this asymmetry-the Froude number ${ }^{12}$ or the surface displacement-has not been clearly determined. ${ }^{13}$ However, in decaying turbulence, several mechanisms could act together (vortex merging, stability, wave vortex interaction), but few studies have been devoted to either homogeneous isotropic turbulence ${ }^{14}$ or the stability of large-scale jets or vortices. Arai and Yamagata ${ }^{13}$ studied numerically the stability and the elongation of an elliptical Gaussian vortex. They found that the cyclonic vortex splits into two vortices, whereas anticyclones always reach axisymmetrization for maximum relative surface deviation $\eta_{\max }$ larger than 0.5-0.6. Stegner and Dritschel ${ }^{15}$ investigated the stability of a generalized isolated shallow-water vortex using a contouradvective semi-Lagrangian algorithm. They show that ageostrophic effects tend to stabilize anticyclones and destabilize cyclones. In addition, they also found, as did Arai and Yamagata, ${ }^{13}$ that the stability of a coherent structure increases when the deformation radius is reduced. A recent study by Poulin and Flierl ${ }^{16}$ has investigated the asymmetry in the destabilization of a Bickley jet. They show that cyclones become more elongated than anticyclones as the Rossby number increases. For stable vortices, a recent work of Graves et al. ${ }^{17}$ has shown that an impulsive strain perturbation induces a greater weakening for cyclones than for anticyclones. Such asymmetry appears for finite Rossby number and deformation radius. Furthermore, when the external strain flow is sustained, the axisymmetric component of anticyclones is strengthen systematically when the defor- 
mation radius is comparable to the vortex radius.

The cyclone-anticyclone asymmetry has also been investigated in a stratified configuration. In such a case, the baroclinic instability could induce a selection between structures of opposite vorticity. Linden et al. ${ }^{18}$ performed laboratory experiments on three-dimensional rotating stratified turbulence and observed a predominance of coherent anticyclones when the deformation radius is smaller than the size of the tank. A cyclone-anticyclone asymmetry was also observed in a numerical study of three-dimensional stratified turbulence for finite Rossby number; ${ }^{19}$ vorticity skewness becomes negative, indicating a dominance of anticyclones. Few numerical studies have addressed the stability of localized vortices in a two-layer model beyond the quasi-geostrophic (QG) regime. Matsuura showed the influence of the surface deviation. ${ }^{20}$ Indeed, large interface displacement is found to stabilize anticyclones and destabilize cyclones. Another study, by Baey and Carton, ${ }^{21}$ on a two-layer shallow-water model showed that cyclones were linearly more unstable than anticyclones when the vortex size becomes larger than the deformation radius.

According to these studies, large-scale flows increase the stability of anticyclonic structures compared to their cyclonic counterpart. Cyclones tend to be more distorted and unstable when their size exceeds the deformation radius. However, this large-scale asymmetry was never studied for a Karman wake. In such a case, both global (the wake) and local (the vortices) structures could be affected.

Hence, the aim of this paper is to study:

- the changes in the Karman wake when it reaches the largescale regime,

- the influence of an expected cyclone-anticyclone asymmetry on the vortex size and the Strouhal number,

- the region of the parameter space where cycloneanticyclone asymmetry occurs.

We performed laboratory experiments under different regimes. The ratio of the deformation radius with the characteristic scale of the obstacle (here, a cylinder of radius $R$ ) was varied from 0.17 up to 3.5. The Rossby number was kept small $(\mathrm{Ro}=0.06-0.2)$, and the Reynolds number was varied from 200 to 1000 . Hence, we investigated the small-scale regime $\left(R / R_{d} \leqslant 1\right)$, the QG regime $\left(R / R_{d}=1\right)$, and the transition to large-scale flows $\left(R / R_{d}>1\right)$.

The experimental setup is described in Sec. II. We present a qualitative characterization of a large-scale wake in Sec. III. Sections IV-VI are devoted to a more quantitative analysis of the different regions of the wake: the shear layer near the obstacle, the detached vortices, and the lee waves, respectively. The influence of large-scale effects on the Strouhal number is discussed in Sec. VII. We discuss and summarize the results in Secs. VIII and IX.

\section{LABORATORY EXPERIMENTS}

\section{A. Experimental setup}

The experiments were performed at the Department of Mechanics UME, ENSTA, in Palaiseau, on a $1.5 \mathrm{~m}$ diameter rotating table (Fig. 1). The upper plate of a turntable rotates
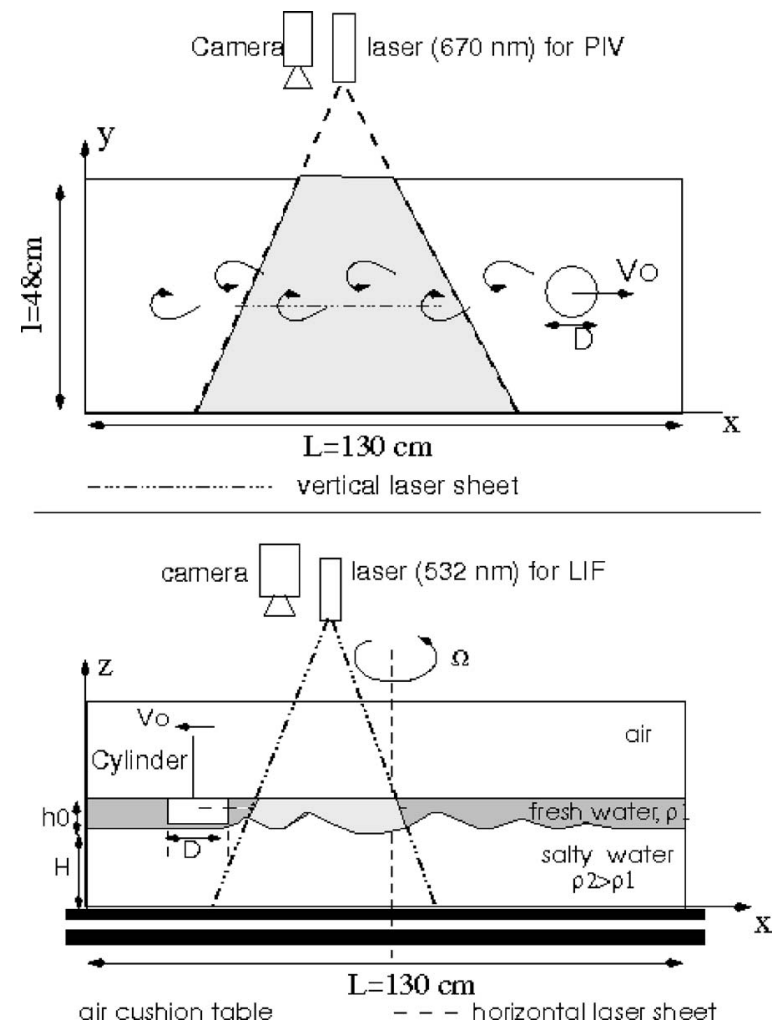

FIG. 1. Top and side views of the experiment. The fluid is stratified in two layers of depth $H=15 \mathrm{~cm}$ and $h_{0}=2 \mathrm{~cm}$. The cylinder is towed in the thin upper layer. The dynamics are observed and recorded by means of a horizontal and a vertical laser sheet.

on a thin air layer in order to reduce friction and mechanical vibrations. We used a rectangular tank, $l=48 \mathrm{~cm}$ wide and $L=130 \mathrm{~cm}$ long. The angular velocity of the turntable $(\Omega)$ was varied from 1 to $12 \mathrm{rpm}$, and the corresponding Coriolis parameter $(f=2 \Omega)$ ranges between 0.1 and $1.25 \mathrm{rad} \mathrm{s}^{-1}$.

We used a two-layer salt stratification that could support either barotropic or baroclinic motions. However, for a total fluid depth $H+h_{0}=17 \mathrm{~cm}$, with $H$ and $h_{0}$ the bottom and upper layer depths, respectively; the barotropic deformation radius $R_{d}=\sqrt{g\left(H+h_{0}\right)} / f$ is as large as the tank $\left(R_{d} \geqslant 1.2 \mathrm{~m}\right)$. Moreover, we adjusted the density difference between the two layers to obtain a small baroclinic deformation radius $R_{d}^{*}=\sqrt{g^{*}\left[h_{0} H /\left(h_{0}+H\right)\right]} / f$, where $h_{0}=2 \mathrm{~cm}$ is the upper layer depth and the reduced gravity $g^{*}=g\left(\rho_{2}-\rho_{1}\right) / \rho_{1}$, with $\rho_{1}$ and $\rho_{2}$ the densities of the upper and bottom layers, respectively. In our case $\left(h_{0}\right.$ small in comparison to $\left.H\right)$, the baroclinic deformation radius can be approximated by $R_{d}^{*} \approx \sqrt{g^{*} h_{0}} / f$ and varied from 1 to $24 \mathrm{~cm}$. We towed a cylinder of diameter $D=7 \mathrm{~cm}$ and height $h_{C}=2 \mathrm{~cm}$ in the upper layer. Hence, we transfer momentum and kinetic energy mainly in the upper layer. The motion in the wake has, therefore, a strong baroclinic component: intense velocities in the thin layer while the deep lower layer remains almost at rest. The deformation of the free surface can be neglected in comparison with the deviation of the internal interface between the two layers. The cylinder diameter may then be equal to or larger than the 
baroclinic deformation radius $R_{d}^{*}$. Therefore, this experimental setup allows us to study the dynamics of large-scale wakes.

To avoid mixing between the two layers, we proceeded as follows. We first filled the tank with salty water to a depth of $H=15 \mathrm{~cm}$. When the solid-body rotation was reached, we slowly injected the light upper layer at the surface of the bottom layer. We then waited until the residual motion of the second layer became negligible, and we towed the obstacle in the upper layer at a constant velocity $V_{0}$ for values varying from 0.28 to $1.5 \mathrm{~cm} \mathrm{~s}^{-1}$.

Standard particle image velocimetry (PIV) was used to measure the horizontal velocity field. Small buoyant particles were put in the upper layer and lightened with a horizontal laser sheet of wavelength $670 \mathrm{~nm}$, located $1 \mathrm{~cm}$ below the upper free surface. The particles' motion was recorded by two $768 \times 576$ pixel $C C D$ cameras rotating with the tank. One used a wide-angle to analyze the global structure of the wake with a resolution of 17 pixels $/ \mathrm{cm}$. The other one is zoomed at a resolution of 45 pixels $/ \mathrm{cm}$ to obtain an accurate resolution of the detached boundary layer just behind the cylinder. The particles' velocity was analyzed using LAVISION PIV software with successive cross-correlation boxes yielding a final $64 \times 48$ vector field for both resolutions. Vorticity fields were derived from velocity fields averaged over a period corresponding to one tenth of a typical turnover time $\tau=D / V_{0}$.

The fluctuations of the internal interface between the upper and the lower layer are measured using a laser-induced fluorescence (LIF) technique. The fluorescent dye (Rhodamine 6G) put in the upper layer was illuminated by a vertical laser sheet. To optimize the fluorescent emission of the Rhodamine $6 \mathrm{G}$, we used a $532 \mathrm{~nm}$ green laser, whose wavelength is close to $530 \mathrm{~nm}$, the ray of maximal absorption of the dye. The fluorescent upper layer appeared bright while the deep lower layer, transparent to the laser sheet, remained dark. A $768 \times 576$ pixel CCD camera is fixed on one side of the tank, perpendicular to the laser sheet. Hence, with this original nonintrusive technique, we were able to measure along a line the displacement of the interface between the light and dense water with an accuracy of $0.5 \mathrm{~mm}$ at an acquisition rate of $25 \mathrm{~Hz}$.

For a few experiments, horizontal PIV measurements and vertical LIF visualizations were used simultaneously. In this case, specific optical filters were fixed on each camera to only detect the dye emission in the vertical plane and the particles in the horizontal plane. We were then able to measure at the same time the horizontal velocity and the upperlayer thickness along one profile. Hence, we could obtain direct measurements of the flow balance and the potential vorticity just behind the cylinder when the flow remained unidirectional.

\section{B. Parameters}

The forcing conditions were fixed by seven physical parameters: the upper layer height $h_{0}$, the reduced gravity $g^{*}$, the cylinder diameter $D=2 R$, the Coriolis parameter $f$, the cylinder velocity $V_{0}$, the fluid viscosity $\nu$, and the bottom- layer depth $H$. Taking $1 / f$ as the reference time scale, we obtain only six independent length scales. Thus, according to dimensional analysis, the dynamical evolution of the wake is governed by five dimensionless parameters. We introduce the Reynolds number Re, the Rossby number Ro, the Burger number $\mathrm{Bu}$, the aspect ratio between vertical and horizontal scales $\alpha$, the layer-thickness ratio $\delta$, and the Ekman number:

$$
\begin{aligned}
& \mathrm{Ro}=\frac{2 V_{0}}{f D}, \quad \mathrm{Re}=\frac{V_{0} D}{\nu}, \quad \mathrm{Bu}=\left(\frac{2 R_{d}^{*}}{D}\right)^{2}, \\
& \alpha=\frac{h_{0}}{D}, \quad \delta=\frac{h_{0}}{H+h_{0}}, \quad \mathrm{Ek}=\frac{\nu}{f H^{2}} .
\end{aligned}
$$

In order to satisfy the geostrophic balance, the Rossby number was kept small $(\mathrm{Ro}=0.06-0.35)$ for all experiments. The Ekman number is about Ek $=7 \times 10^{-5}$; therefore, Ekman pumping is very weak in the bottom layer and will not affect the dynamics of the upper layer. Besides, due to the small aspect ratio $(\alpha=0.28)$ and according to the condition $\alpha^{2}$ Ro $\ll 1,{ }^{22}$ the hydrostatic balance is verified in the upper layer. Moreover, we checked that the lower layer velocity $V_{\text {bottom }}$ $\simeq \alpha V_{0}$ remained small compared to the upper layer velocity $V_{0}$. Hence, even if the lower-layer aspect ratio $\left(\alpha_{\text {bottom }}\right.$ $=H / D=2.14)$ is not negligible, the relation $\alpha_{\text {bottom }}^{2} \mathrm{Ro}_{\text {bottom }}$ $\ll 1$, where $\mathrm{Ro}_{\text {bottom }}=V_{\text {bottom }} / f R$, guarantees that the hydrostatic approximation is also respected in the bottom layer. Therefore, the vertical variations of horizontal velocities remain small and we can expect shallow-water dynamics in both layers. The layer-thickness ratio $\delta$ controls the dynamical interactions between the two layers. According to Cushman-Roisin, ${ }^{23}$ the upper layer is not affected (to the first order of approximation) by the lower-layer dynamics if $\delta$ $\ll \min \left(1, \mathrm{Bu}^{2}\right)$. This criterion was respected in most of our experiments, as long as the Burger number was not too small. Hence, the upper layer motion could be described by the shallow-water reduced-gravity model. ${ }^{22}$ In that context, the dynamical evolution of the wake is mainly governed by the Reynolds Re, the Rossby Ro, and the Burger $\mathrm{Bu}$ numbers.

We performed experiments for four different Reynolds numbers: $\operatorname{Re}=200,400,800$, and 1000. For these values, periodic vortex shedding always occurred in twodimensional wakes. ${ }^{24,25}$ Besides, in the absence of rotation a secondary three-dimensional instability occurs when Re exceeds $180 .^{26,27}$ Nevertheless, in our case, due to the rotation and the shallow-water configuration, we did not see any evidence of such three-dimensional instabilities.

The Burger number was varied over a wide range of values: $\mathrm{Bu}=0.11-46$. Small Burger numbers $(\mathrm{Bu}=0.11-1)$ characterize large-scale flows $\left(R_{d}^{*} \leqslant R\right)$, while $\mathrm{Bu} \gg 1$ correspond to small-scale flows in which the influence of rotation could be neglected in the limit of small Rossby number. The use of the Burger number is not generalized in the literature and one could choose other dimensionless number to characterize the flow regime. Hence, we introduced the relative interface deviation $\lambda$ and the Froude number Fr: 


$$
\lambda=\frac{\mathrm{Ro}}{\mathrm{Bu}}, \quad \mathrm{Fr}=\frac{V_{0}}{\sqrt{g^{*} h_{0}}}=\frac{\mathrm{Ro}}{\sqrt{\mathrm{Bu}}} .
$$

For small Rossby numbers, the flow satisfies the geostrophic balance at first order. This balance forced the parameter $\lambda$ to be related to the relative interface deviation: $\lambda \propto \eta=\Delta h_{0} / h_{0}$. In what follows, $\mathrm{Bu}, \mathrm{Fr}$, or $\lambda$ is used to quantify the transition from a small- to large-scale regime.

\section{DYNAMICAL STRUCTURE OF THE WAKES}

To make a qualitative comparison between small-scale and large-scale wakes, we show in Fig. 2 the evolution of vorticity fields in two extreme cases. The first case [Figs. 2(a)-2(c) ] corresponds to a $\mathrm{QG}$ regime: $\mathrm{Ro}=0.06, \mathrm{Bu}=1.97$, and $\mathrm{Re}=196$. In this case, the deformation radius is comparable to the cylinder radius. According to Fig. 2(a), strong velocity gradients occur in the boundary layers around the cylinder. The boundary layers then roll up alternatively on each side of the cylinder generating a periodic succession of elliptical vortices. Further down the wake, these vortices become more circular. Cyclones and anticyclones present the same dynamical evolution. This scenario is identical to the formation of a classical two-dimensional Karman street. ${ }^{26}$ The second case [Figs. 2(d)-2(f)] corresponds to a largescale wake: $\mathrm{Ro}=0.19, \mathrm{Bu}=0.11$, and $\mathrm{Re}=798$. According to Fig. 2(d), the boundary layers formed on the cylinder detach but do not roll up immediately. Instead, the wake behind the cylinder exhibits cyclonic (in red) and anticyclonic (in blue) shear layers which remain stable up to a distance of two to three diameters. Further down, the wake becomes unstable, anticyclonic vortices are formed but no coherent cyclonic structures emerged. The instability mechanism in that case is not easy to identify: is it wake instability or shear instability? In this configuration the wake exhibits a strong asymmetry between the cyclonic and anticyclonic regions. Due to the absence of coherent cyclones, the distance separating anticyclones is reduced in the large-scale regime. While four anticyclones are observed in the large-scale wake [Fig. 2(f)], only two appear in the QG wake [Fig. 2(c)] for the same resolution. Therefore, the shedding frequency is twice as important in the large-scale configuration as in the QG regime. Such an increase in the shedding frequency is much more important than the variations observed for nonrotating and rotating Karman streets when varying the Reynolds and Rossby numbers. ${ }^{28}$ Hence, the wake in the large-scale configuration is strongly asymmetric and the frequency vortex shedding is significantly increased.

\section{SHEAR LAYER}

To characterize more accurately the flow around the obstacle, we looked at the streamwise velocity profile just behind the cylinder. We plotted the profiles for three different cases in Fig. 3. The first one $(\mathrm{Ro}=0.06, \mathrm{Bu}=0.68, \mathrm{Re}=196$, $\lambda=0.09$ ) corresponds to a $\mathrm{QG}$ regime, and the other two $(\mathrm{Ro}=0.08, \mathrm{Re}=400, \mathrm{Bu}=0.11, \lambda=0.71$; and $\mathrm{Ro}=0.19, \mathrm{Re}$ $=798, \mathrm{Bu}=0.11, \lambda=1.71)$ correspond to large-scale configurations. The horizontal velocity profiles, measured along a spanwise section, are averaged over time to filter out wave

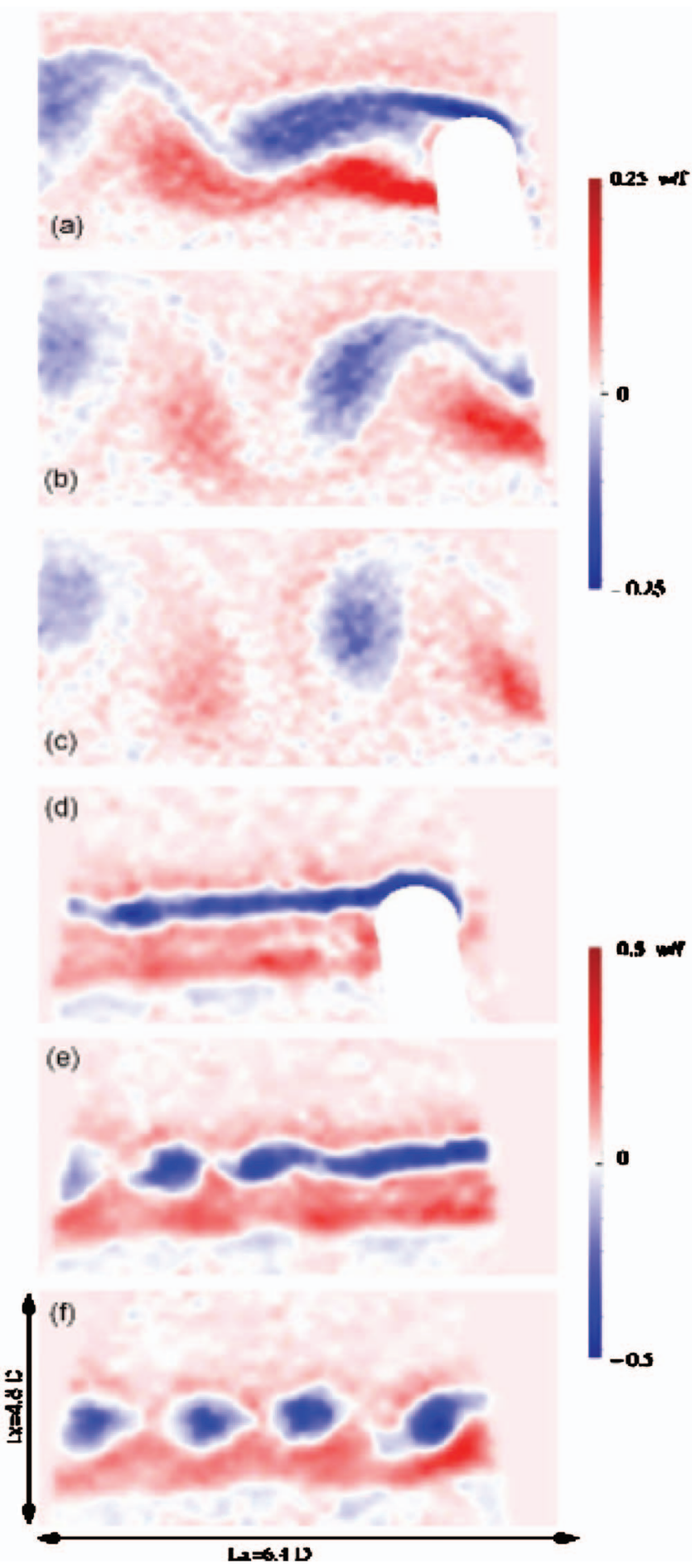

FIG. 2. (Color) Vorticity fields for a small-scale configuration (expt. no. 4) at $T=0 \tau(\mathrm{a}), 3 \tau(\mathrm{b})$, and $5.2 \tau(\mathrm{c})$, and a large-scale configuration (expt. no. 23) at $T=0 \tau(\mathrm{d}), 2.4 \tau(\mathrm{e})$, and $5.5 \tau(\mathrm{f})$, where $\tau=D / V_{0}$ is the vortex turnover time. The cylinder and its shadow in the laser sheet are put in white on images (a) and (d). Blue corresponds to anticyclonic and red to cyclonic vorticity.

motions (see Sec. VI). The QG profile shows a symmetrical wake. As the interface deviation increases, the intensity of the cyclonic shear layer decreases, leading to a highly asymmetric velocity profile (Fig. 3 for $\lambda=1.7$ ). Hence, in a largescale configuration, the flow around the obstacle loses its symmetry. 


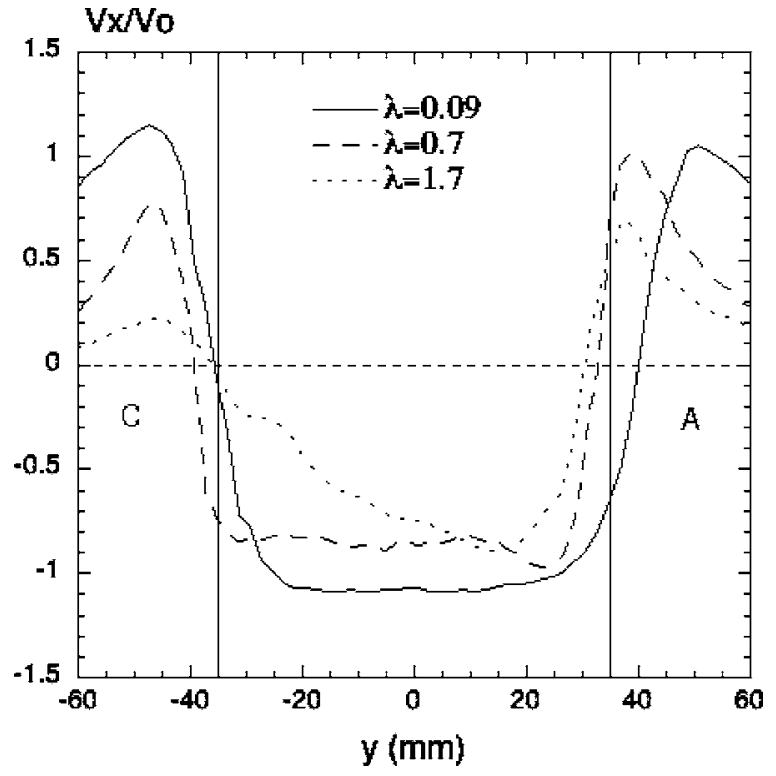

FIG. 3. Dimensionless streamwise velocity profiles behind the cylinder for different parameters (the solid line corresponds to expt. no. 10, the dashed line to expt. no. 22, and dot line to expt. no. 23). Letters "A" and "C" refer to the cyclonic and anticyclonic parts of the shear layer, respectively. The vertical lines indicate the diameter of the cylinder.

To quantify this asymmetry, we looked at the vorticity difference between the anticyclonic and cyclonic shear layers just behind the cylinder. According to Fig. 4, this vorticity difference increases with the interface deviation, independent of the Reynolds number. For a small surface displacement, vorticity has the same value in each shear layer, which corresponds to a symmetric flow. For an interface deviation larger than 0.3 , cyclonic vorticity becomes smaller than the anticyclonic vorticity. Although the vorticity difference seems to be independent of the Reynolds number, the shear layer vorticity do increase with the Reynolds number. When this latter is about 800 , the vorticity $\omega$ in the anticyclonic

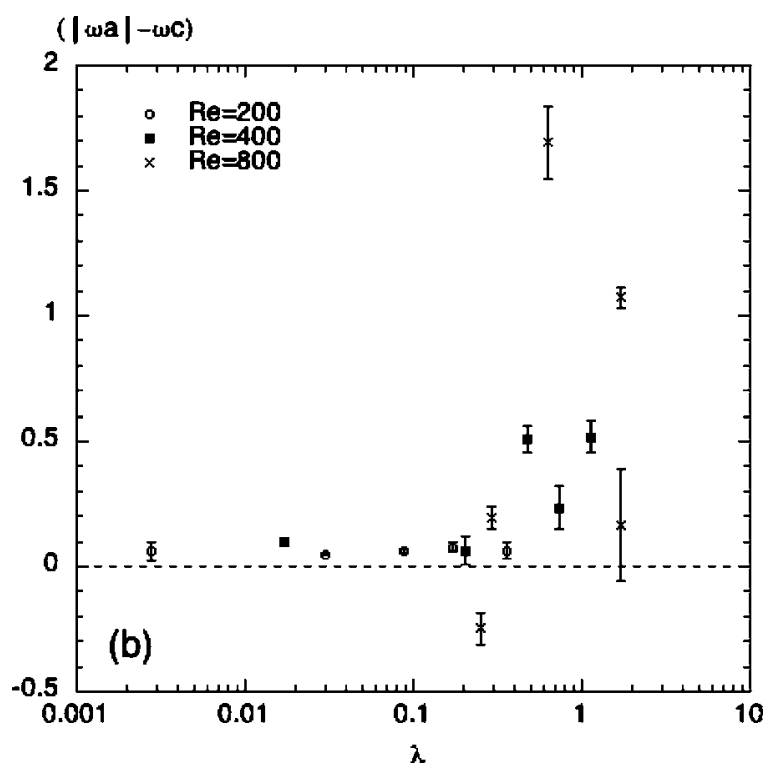

FIG. 4. Vorticity difference between anticyclonic and cyclonic shear layers for all experiments as a function of the relative interface deviation. shear layer is about $-0.8 f$, where $f$ is the Coriolis parameter, and can reach values down to $-1.2 f$. Hence, according to the Rayleigh criteria for inertial instability ${ }^{29,30}(\omega \leqslant-f)$, the anticyclonic parallel shear flow could be unstable. Yet, we do not observe any evidence of three-dimensional instabilities in this region. This could be due to the shallow water configuration which restricts vertical motions. However, for larger Reynolds numbers, inertial instability could affect the region of negative vorticity.

Kuo and Polvani showed that the geostrophic adjustment of an unbalanced velocity field could induce an asymmetry between cyclonic and anticyclonic vorticity regions. ${ }^{31}$ Such asymmetry is found to be enhanced for large-scale flows. Hence, one could ask whether the mean flow is in geostrophic balance. We then measured simultaneously the velocity and the interface displacement behind the obstacle for a large-scale wake configuration: $\lambda=1, \mathrm{Bu}=0.24, \mathrm{Ro}=0.24$, and $\operatorname{Re}=1000$. The double laser sheet setup described above was necessary to obtain quantitative measurements of the horizontal velocity and interface deviation profiles plotted in Fig. 5 (dots). As in Fig. 3, the velocity profile is averaged with time to filter out the fast wave motion (see Sec. VI). To estimate the derivative or the integral of the mean measured velocity profile, we fitted the discrete measurements with a smoothed profile using a Fourier low-pass filter. Using this continuous velocity fit, we calculated the mean geostrophic geopotential height using

$$
f u_{\mathrm{fit}}=g^{*} \frac{\partial \eta_{\mathrm{geo}}}{\partial y} .
$$

For this range of parameters the mean interface deviation has finite amplitude values [Fig. 5(b)] and the anticyclonic high pressure $\left(\Delta p=0.4 \rho g^{*} h_{0}\right)$ is twice the cyclonic low pressure $\left(\Delta p=-0.2 \rho g^{*} h_{0}\right)$. Besides, according to Fig. 5(b), the mean geopotential is in geostrophic balance with the mean velocity in the whole central region. Nevertheless, one observes some discrepancies between $h_{\text {geo }}$ and $h_{\text {mes }}$ at the periphery. Therefore, we cannot exclude a low fraction of unbalanced motion behind the cylinder. The mean geostrophic vorticity and potential vorticity profiles are then derived from the following relation:

$$
q_{\mathrm{geo}}=\frac{\omega_{\mathrm{geo}}+1}{\eta_{\mathrm{geo}}+1}-1
$$

where $\omega_{\text {geo }}=\left(g^{*} / f\right)\left(\partial^{2} \eta_{\text {geo }} / \partial y^{2}\right)$. These profiles are compared to the mean measured profiles of vorticity and interface deviation. Experimental mean potential vorticity is then deduced from

$$
q_{\exp }=\frac{\omega_{\exp }+1}{\eta_{\exp }+1}-1
$$

Figure 5 shows that the geostrophic fit is in good agreement with the mean measured potential vorticity. Hence, according to these simultaneous measurements of the horizontal velocity and the interface deviation, the time-averaged unidirectional shear just behind the cylinder remains close to 

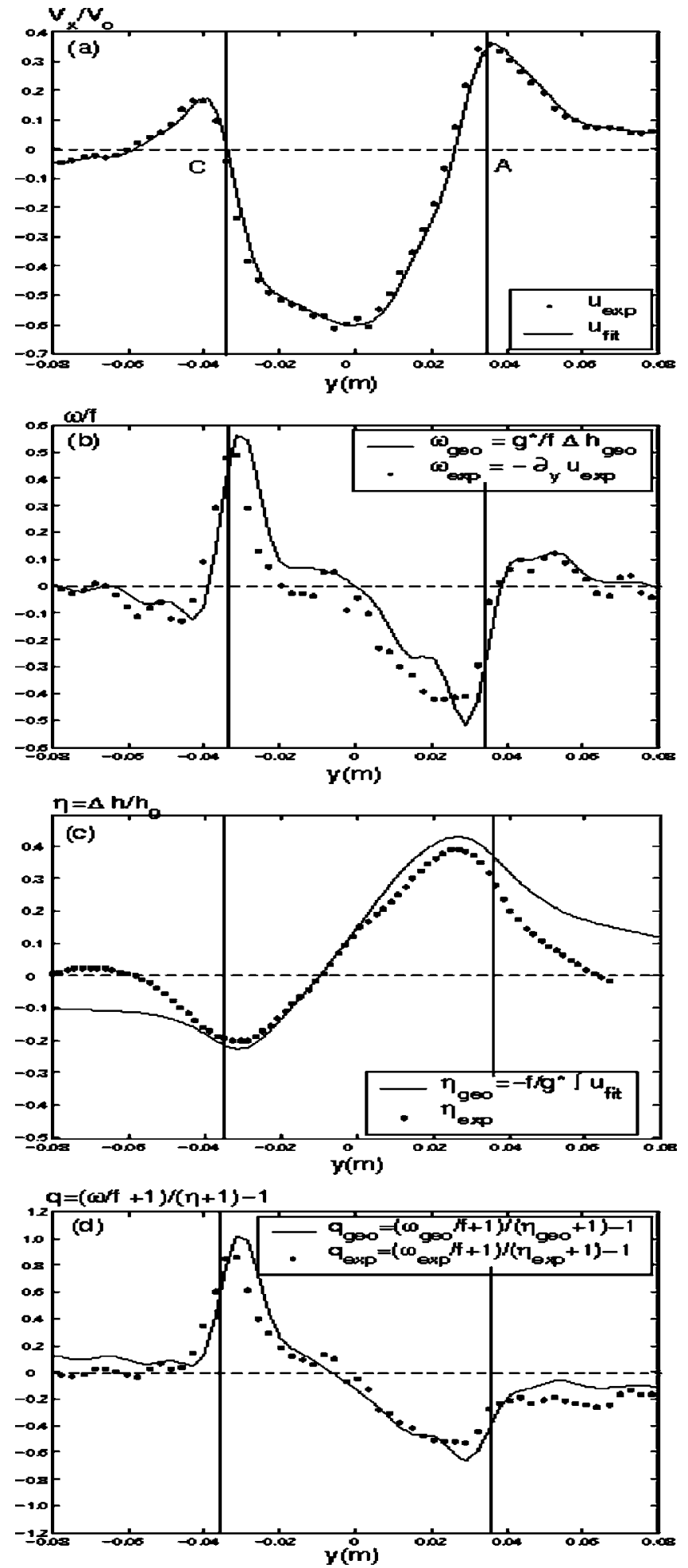

FIG. 5. (a) Measured (dots) and fitted (solid line) profiles of velocity; (b) profiles of vorticity derived from experimental (dots) and fitted (solid line) velocity profile; (c) measured (dots) relative interface deviation and in geostrophic balance with the fitted velocity profile (solid line); (d) potential vorticity profiles calculated from experimental profiles of vorticity and interface deviation (dots) and profiles of vorticity and interface deviation in geostrophic balance (solid line) for expt. no. 20.

geostrophic balance. Besides, in the large-scale regime all dynamical fields $(h, \omega, q)$ for this mean shear flow present a significant asymmetry between cyclonic and anticyclonic vorticity regions.

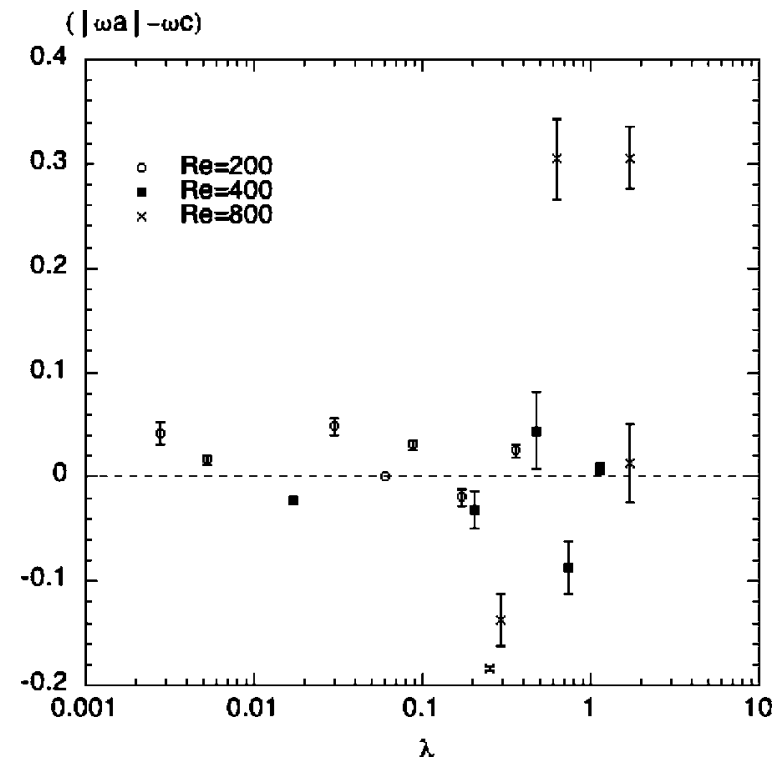

FIG. 6. Difference of vorticity between the first detached anticyclone and the first detached cyclone, averaged over a small area around the center of vortices, as a function of the relative interface deviation.

\section{VORTICES}

The size and the intensity of coherent vortices formed in standard Karman streets are controlled by the obstacle diameter and the flow velocity. In the large-scale regime one could ask how the deformation radius impacts on the vortices formed in the wake. We first studied the intensity, i.e., the vorticity, of these vortices. We plotted in Fig. 6 the vorticity difference between the first detached anticyclone and cyclone, averaged over a small area in the center of the vortex. When no coherent cyclone appeared, we considered the vorticity, although not coherent, in the cyclonic counterpart of the first coherent anticyclone. The relative interface deviation was used here to characterize the transition from small-scale to large-scale parameters. For a small interface deviation, the vortex intensity difference remained almost constant and close to zero. For an interface deviation larger than about 0.2 , the vorticity intensity difference increased. However, the vorticity difference is either positive or negative, and the maximum vorticity difference in the detached vortices is three times less than in the shear layer. The dissipation tends to attenuate the intensity of the asymmetry in the vortices.

We then studied the impact of the deformation radius on the vortex size. We used the transverse velocity profile to estimate the size of the vortices. We then averaged the halfdistance between the minimum and maximum velocity for each structure. One could expect the size of the vortices to scale with the deformation radius $R_{d}$ when it becomes smaller than $R$, the radius of the cylinder. For small-scale cylinders $\left(R \leqslant R_{d}\right)$, geopotential deviation should not affect the wake and the vortices will then scale only with $R$. Indeed, according to Fig. 7, we found that at small scales $\left(R \leqslant R_{d}\right)$, the radius of the vortices remained constant. Vortices scale with the size of the obstacle, as for the classical twodimensional case. For a deformation radius smaller than the size of the cylinder $\left(R \geqslant R_{d}\right)$, nearly all vortical radii become 


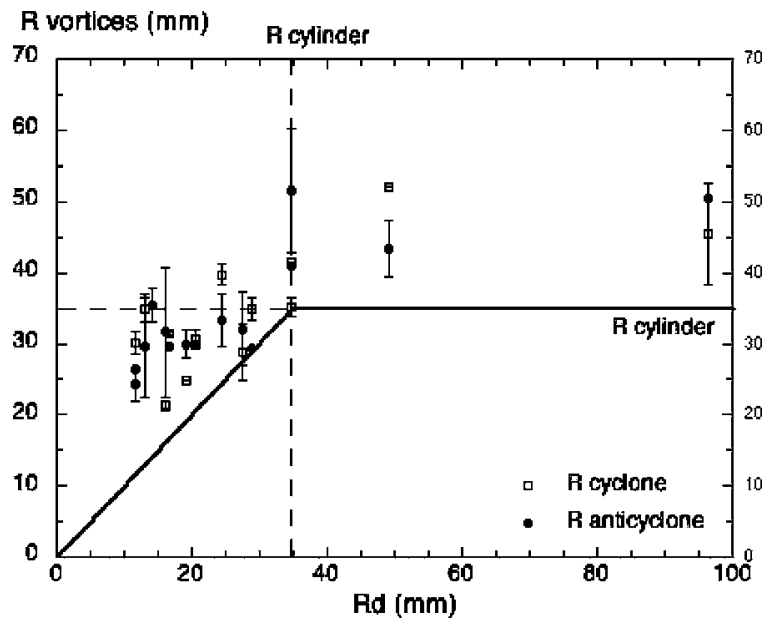

FIG. 7. Radius of anticyclones $(\bullet)$ and cyclones $(\square)$ in the vortex street as a function of the deformation radius. The dashed lines indicate the radius of the cylinder $R$. The solid line shows the separation between radii larger or smaller than the deformation radius.

smaller than the obstacle, but remain larger than the deformation radius. When the wake enters in the large-scale regime, the vortex size is reduced but does not exactly scale with $R_{d}$.

\section{WAVE ACTIVITY}

The rotating two-layer system may support both internal gravity waves and inertial waves. Hence, in our experiments for a subcritical regime, the rigid obstacle could be strongly coupled to nonhydrostatic wave components. In the nonrotating case, the generation of internal waves in the lee of an obstacle is maximum when the Froude number is close to but smaller than unity. In the rotating case, the situation could be more complex.

We detected significant wave motion only in the largescale regime when the Froude number increases up to 0.58 . Showing a side view of the upper layer (Fig. 8), we observe
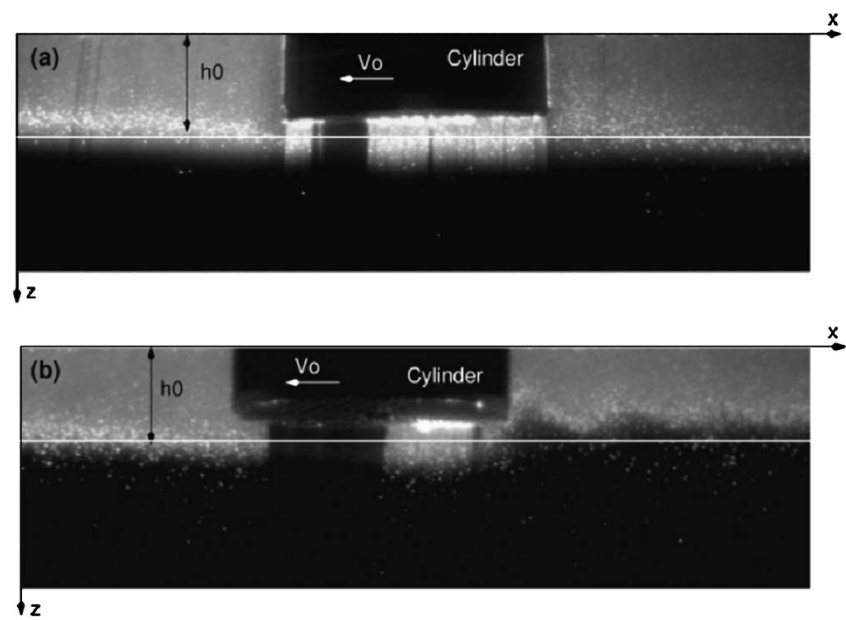

FIG. 8. Side view of the upper layer, in light gray on the images. The slice is oriented streamwise in the anticyclonic part of the wake for expt. no. 9, corresponding to a QG regime (a) and in the cyclonic part of the wake for expt. no. 23 , corresponding to a frontal geostrophic regime (b).

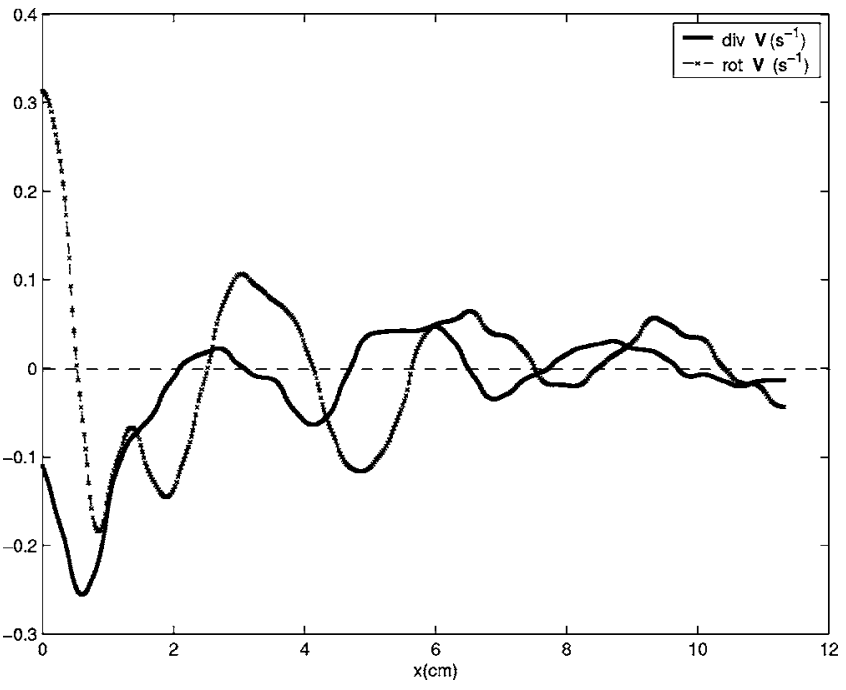

FIG. 9. Spanwise averaged slice of divergence (solid line) and vorticity (dashed line) fields of expt. no. 23.

that the interface remained flat in the QG regime $\left(R=R_{d}\right)$, while small-wavelength disturbances appeared in the largescale regime $\left(R=5 R_{d}\right)$. We do not observe a sudden apparition of the waves at a critical Froude number. Nevertheless, due to the fact that their wavelength are comparable to the layer thickness, we suspect these waves to be associated with nonhydrostatic motion. In such case, the waves are dispersive and subcritical and their amplitude is expected to increase gradually with the Froude number. Further down in the wake, the waves are rapidly smoothed and vortices are formed. The waves were attached to the moving obstacle, and they can reach a strong amplitude up to $40 \%$ of the upper-layer thickness. However, this wave activity remained confined just behind the cylinder, and we could hardly detect more than three or four oscillations in this region. We plotted in Fig. 9, the vorticity and the divergence profile along the streamwise direction behind the cylinder, for $\mathrm{Bu}=0.11$, Ro $=0.19$, and $\mathrm{Re}=798$. These profiles were spatially averaged along the spanwise direction in the cyclonic vorticity region. The wave motion was confirmed by the observation of a significant divergence field which tend to be in phase quadrature with the vorticity field. The characteristics of these waves are given in Table I for three cases. According to our measurements, the wave period (observed in the frame of the rotating tank) was close to the inertial period $\left(T_{f}=2 \pi / f\right.$ $=3.75 \mathrm{~s}$ ). Hence, in the large-scale regime, the rigid obstacle tended to generate inertial waves. This wave field also presented a cyclone-anticyclone asymmetry. Indeed, the layer thickness was smaller in the cyclonic part of the wake compared to its anticyclonic part. For a large-scale regime, this difference became significant. Therefore, the local Froude number was higher in the cyclonic shear layer. This could explain the stronger wave activity observed in this region.

\section{STROUHAL NUMBER}

We used the overhead view and PIV measurements to estimate the influence of the cyclone-anticyclone asymmetry on the Strouhal number. The Strouhal number is usually de- 
TABLE I. Characteristics of the waves observed behind the cylinder for experiment numbers 19, 20, and 23. The parameter $\lambda$ is the interface deviation, Fr the Froude number, and $R_{d}$ the deformation radius; $\lambda_{w}$, the wavelength; $T_{w}$, the wave period; $V_{0}$, the obstacle velocity; $c$, the wave velocity; $A$, the wave amplitude; and $h_{0}$, the layer depth.

\begin{tabular}{ccccccc}
\hline \hline Fr & $\lambda$ & $T_{w}(\mathrm{~s})$ & $V_{0} / c$ & $A / h_{0}$ & $\lambda_{w}(\mathrm{~cm})$ & $R_{d}(\mathrm{~cm})$ \\
\hline 0.41 & 0.90 & 2.16 & 1.00 & 0.24 & 2.25 & 1.60 \\
0.44 & 1.06 & 2.16 & 1.02 & 0.40 & 2.36 & 1.43 \\
0.58 & 1.71 & 3.84 & 0.90 & 0.40 & 3.00 & 1.17 \\
\hline \hline
\end{tabular}

fined as the dimensionless shedding frequency: $\mathrm{St}=F D / V_{0}$, where $F$ is the shedding frequency of one-sign vortices, $D$ is the size of the obstacle, and $V_{0}$ its velocity. We used a geometrical method considering a small number of vortices to estimate $\mathrm{St}=\left(1-V_{d} / V_{0}\right) D / L$, where $V_{d}$ is the drift velocity of the Karman street and $L$ the average distance between successive vortices of the same sign.

For the cases in which no cyclones appear, the Strouhal number was calculated considering only the shedding frequency of anticyclones. Figure 10 shows the dimensionless shedding frequency for different Reynolds numbers, as a function of Burger number, Froude number, and interface deviation. The most striking result is that the Strouhal number reaches very large values compared to common values found in the literature for non rotating as well as rotating flows. ${ }^{26,32}$ In nonrotating dynamics, the Strouhal number is a function of Reynolds number only and never exceeds $0.24 .^{24,26}$ The range of Strouhal numbers for Reynolds between 200 and 1000 is indicated by the dashed zone on Fig. 10 ; it varies between 0.2 and $0.24{ }^{24}$ In rotating flow, the shedding frequency increases weakly for strong rotation; i.e., for small Rossby number. This trend was observed by Boyer et $a l^{33,34}$ for large Reynolds numbers and by Stegner et al. ${ }^{35}$ for moderate Reynolds numbers, but nevertheless in both studies the measured values remain very close to the twodimensional nonrotating case.

Hence, the strong increase in the Strouhal number is due mainly to the large-scale configuration. According to Fig. 2 when the size of the obstacle becomes larger than the deformation radius, the distance between anticyclones is reduced due to the disappearance of coherent cyclones. This simple fact explains the strong increase in the shedding frequency. Thus, the Strouhal number appears to be an efficient parameter to quantify the cyclone-anticyclone asymmetry of the wake. On one hand, we plotted the Strouhal number as function of Burger number [Fig. 10(a)]. The values are very scattered, and no clear trend emerged in the large-scale region $(\mathrm{Bu} \leqslant 1)$. Hence, the ratio between the deformation radius and the size of the obstacle is not the accurate parameter to quantify the cyclone-anticyclone asymmetry. On the other hand, we plotted the Strouhal number as a function of the Froude number or the interface deviation parameter [Figs. 10(b) and 10(c)], all points seem to collapse on the same curve, for various values of the Reynolds or the Rossby number. Figure 11 shows the Strouhal number as a function of Reynolds number for different values of the surface deviation $\lambda$. In that range of parameters, for a fixed value of $\lambda$, the Strouhal number remains almost constant, while the Rey- nolds number varies from 200 to 1000 . For instance, when $\lambda \approx 0.2-0.3(\lambda \approx 0.6-0.7)$, the Strouhal number is about 0.3 (0.4). Hence, the Reynolds number seems to have a very weak influence on the shedding frequency and on the vortex asymmetry. For a given Reynolds number, the vortex street may be symmetric (for $\lambda=0.1$ ) as well as asymmetric (for $\lambda=1.0$ ). However, for a smaller Reynolds number we could expect a diminution of the Strouhal number, as in twodimensional incompressible dynamics. In the range of Reynolds and Rossby numbers we studied, either the Froude number or the interface deviation appears to be the accurate controlling parameter of the shedding frequency. To make a clear distinction between these two parameters, data are needed for a wider range of the parameters. This would imply variations in the Burger number between 0.01 up to 1 .

\section{DISCUSSION}

We have determined that a single parameter controls the cyclone-anticyclone asymmetry of the wake. This asymmetry occurs when the relative interface deviation parameter $\lambda$ exceeds $0.2-0.3$. According to Fig. 12, showing the relation between the effective surface deviation $\eta=\Delta h / h_{0}$ and the parameter $\lambda=\mathrm{Ro} / \mathrm{Bu}$, this threshold corresponds to relative geopotential deviations of $30 \%$.

Considering a rotating shallow-water model, we could understand the influence of the finite geopotential deviation. According to the geostrophic balance, cyclones correspond to troughs in the fluid layer, whereas anticyclones correspond to crests. Therefore, when the size of the vortices increases the geopotential deviation also increases. Hence, cyclones are limited by the mean geopotential height, whereas anticyclones are not. Large-scale cyclones corresponding to a negative geopotential height cannot exist in this model.

Nevertheless, the asymmetry occurs for intermediate values of $\eta$ that remain below unity (Fig. 12). We should then understand how the stability of the flow may change when the geopotential deviation increases and if selective destabilization may occur. As explained in Sec. II B, the reduced-gravity model describes the dynamics of the upper layer consistently. For the range of parameters we explored, i.e., small Rossby numbers, we filter out the fast wave motions assuming that they have a weak influence on the wake destabilization. In this case, we can use asymptotic intermediate models to study the wake stability. The first model corresponds to the classical QG model: ${ }^{22}$ 


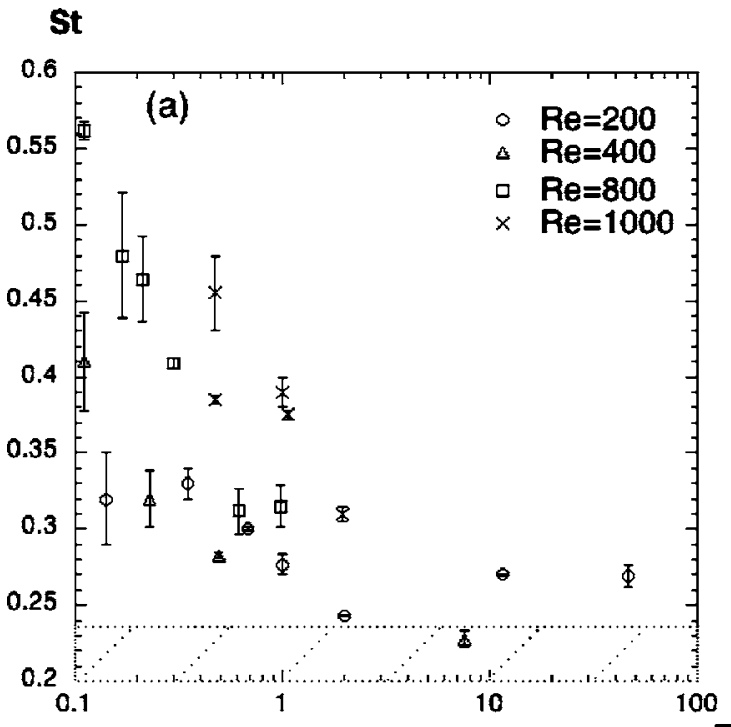

Bu

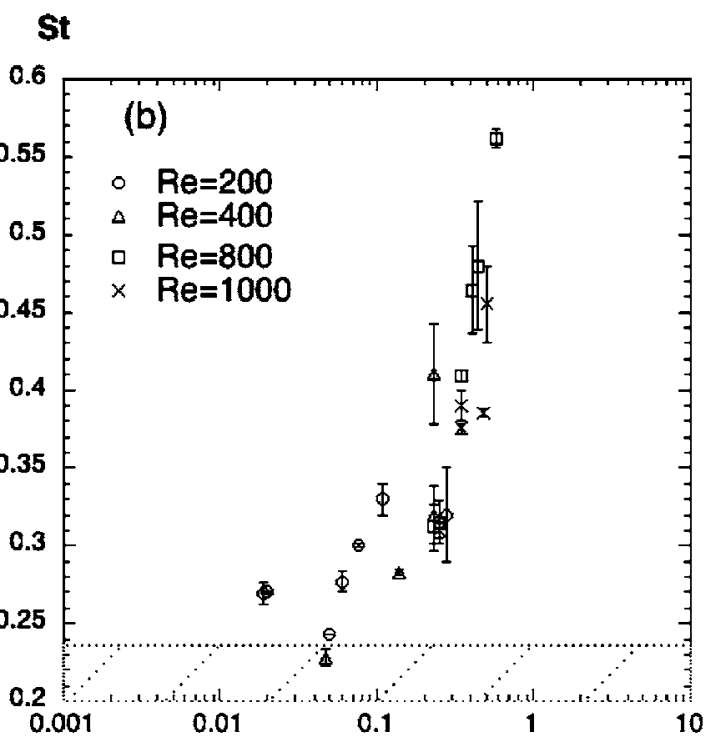

Fr

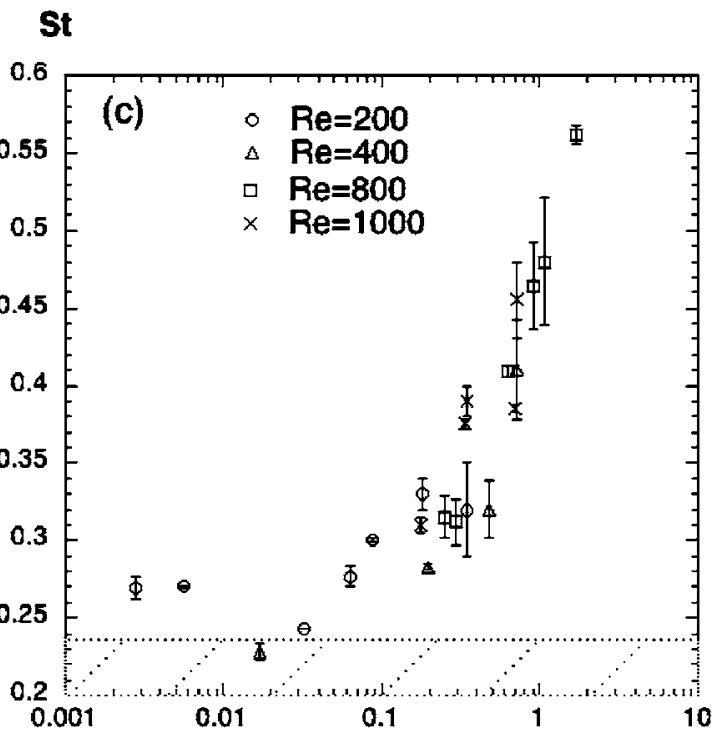

FIG 10. Strouhal number as a function of the Burger number (a), the Froude number (b), and the relative interface deviation (c), plot for different Reynolds numbers. The dashed zone indicates the range of the Strouhal number for a three-dimensional wake at these Reynolds numbers.

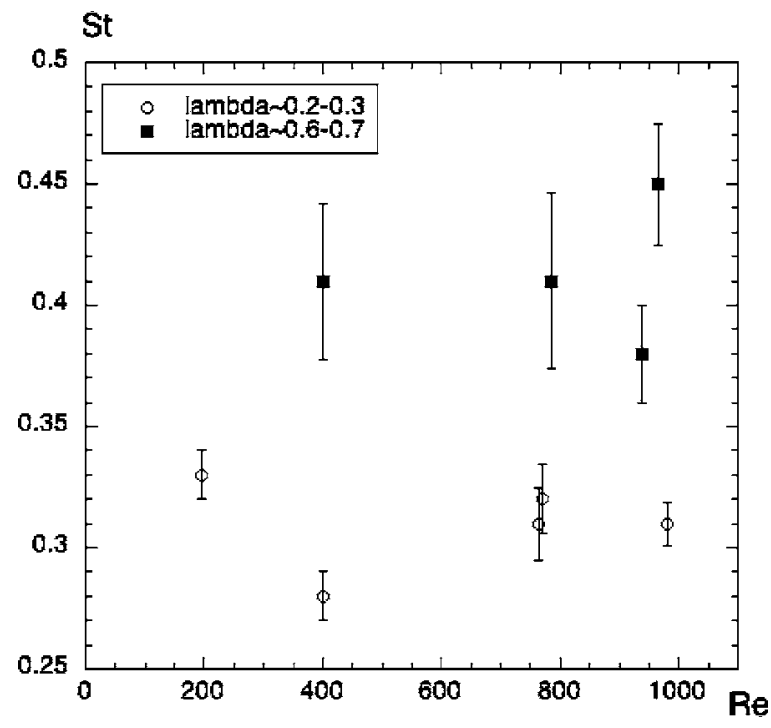

FIG. 11. Strouhal number as a function of the Reynolds numbers for various relative surface deviation $\lambda$.

$$
\frac{\partial}{\partial t}\left(\nabla^{2} \eta-\eta\right)+J\left(\eta, \nabla^{2} \eta-\eta\right)=0
$$

where $J(a, b)=\partial_{x} a \partial_{y} b-\partial_{y} a \partial_{x} b$ is the Jacobian operator. The $\mathrm{QG}$ regime is valid for Burger numbers of order one, which means small surface deviations $(\lambda=\mathrm{Ro} / \mathrm{Bu} \ll 1)$ since Ro $\ll 1$. In this regime, due to the symmetry of the equations, no cyclone-anticyclone asymmetry occurs.

The second model correspond to the frontal geostrophic (FG) model established by Cushman-Roisin: ${ }^{36}$

$$
\frac{\partial \eta}{\partial t}-J\left[\eta,(1+\lambda \eta) \nabla^{2} \eta+\frac{1}{2}(\nabla \eta)^{2}\right]=0,
$$

which holds for length scales much larger than the deformation radius; i.e., $\mathrm{Bu} \leqslant \mathrm{Ro}$ and $\lambda \approx \mathcal{O}(1)$. The cubic terms

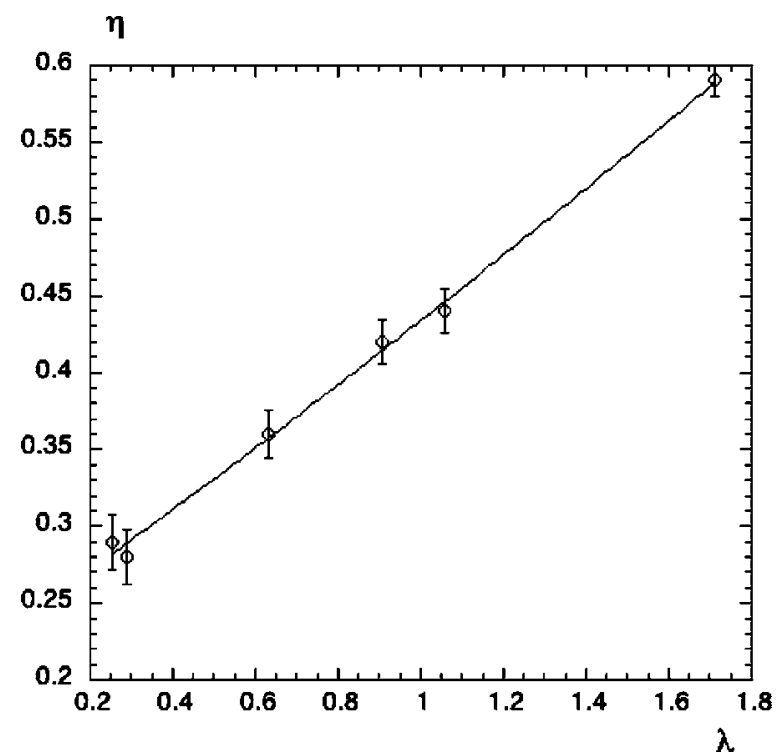

FIG. 12. Maximum geopotential deviation $\eta=\Delta h_{0} / h_{0}$, measured from the side view, as a function of the interface deviation parameter $\lambda=\mathrm{Ro} / \mathrm{Bu}$ 
$J\left[\eta,(1+\lambda \eta) \nabla^{2} \eta+(1 / 2)(\nabla \eta)^{2}\right]$ induce necessarily an asymmetric wake.

Therefore, the shear layer may be destabilized differently in its cyclonic and anticyclonic parts. According to the Rayleigh criterion extended to a FG regime, the flow is linearly stable for a monotonic geopotential profile. Nonlinear stability has also been studied with a Hamiltonian formulation by Holm et al. ${ }^{37}$ and Ben Jelloul and Zeitlin ${ }^{38}$ for localized vortices. Nevertheless, according to this criterion the shear layers of both signs could be unstable [Figs. 5(c) and 5(d)]. To detect an asymmetric destabilization between the cyclonic and anticyclonic parts of the wake, a complete linear stability analysis is then needed.

However, according to our experiments, the anticyclonic shear region leads to stronger vortices. Hence, the anticyclonic shear seems to be more unstable than the cyclonic one for strong geopotential deviation. In such a case, the wake instability would favor the formation of large-scale anticyclonic vortices. Once they are formed these anticyclones are expected to be more robust than their cyclonic counterparts. Indeed, for localized vortices, Arai and Yamagata ${ }^{13}$ and Stegner and Dritschel ${ }^{15}$ found that the unstable growth rate decreases when the vortex size becomes larger than the deformation radius. Besides, the anticyclones are favored in this restabilization process. For stable vortices having monotonic vorticity profiles, Graves, McWilliams, and Montgomery ${ }^{17}$ reveal a new mechanism for dominance of strong anticyclones. They have shown that cyclones, once submitted to an external strain field, weaken strongly, while anticyclones strengthen systematically when the deformation radius is comparable to the vortex radius. Hence, in a large-scale regime, the strong distortion of cyclones observed in our experiments could be due to the strain induced by the anticyclones.

Therefore, several effects could induce a selection of anticyclonic structures in the vortex street: the asymmetric flow splitting around large-scale obstacles, a selective destabilization of parallel wake flows in the frontal regime, and vortexstrain interaction. In order to quantify the relative influence of these mechanisms, numerical studies are under progress and will be presented in a forthcoming paper.

\section{CONCLUSION}

We investigated the dynamics of large-scale Karman streets in the wake of a cylinder towed in a rotating shallowwater layer. According to our measurements, the wake downstream the obstacle satisfies first-order geostrophic balance with small Rossby numbers, while the relative interface deviation reaches values up to $60 \%$ of the layer thickness. Hence, we studied the vortex street formation over a large range of parameter values varying from quasi-geostrophic ${ }^{22}$ to frontal geostrophic regimes. ${ }^{36}$ When the relative geopotential deviation exceeds $30 \%$, a significant asymmetry occurs between cyclonic and anticyclonic vortices in the wake. Large-scale anticyclones remain coherent and circular, whereas cyclones tend to be more elongated and distorted. More surprisingly, for the extreme case of strong interface deviation, only an anticyclonic vortex street emerged in the lee of the obstacle. In this dynamical regime, the wake looks more like an unstable shear layer than a Karman street. Another characteristic of this large-scale regime is the formation of an extended double shear layer, which remains stable for several diameters downstream the cylinder. The vortex street then develops away from the obstacle and the shedding occurs far downstream. We recover in this case some properties of the frontal geostrophic regime: the stabilization of the barotropic shear instability and the asymmetry between cyclones and anticyclones. The main consequence of the distortion or the disappearance of the cyclonic street is a strong increase in the Strouhal number. Indeed, the latter can be three times larger than the standard value reached for the same Reynolds number in a classical Karman street. We found that this variation of the Strouhal number and the cyclone-anticyclone asymmetry is governed by a single parameter: the relative interface deviation $\lambda$. For small values of $\lambda$ the shedding frequency is identical to its classical value in two dimensional flows. ${ }^{24}$ The increase in Strouhal starts when the relative surface deviation $\lambda$ exceeds 0.1 .

This paper may contribute to a better understanding of the formation of large-scale vortices in the ocean behind an island or off a cape. We can expect dominance of anticyclonic vortices when an oceanic or coastal current encounters a large-scale island, although the experimental setting is not equivalent, since the obstacle is moving with respect to the rotating referential, which is not the case of an island. Other phenomena should also be taken into account for a more realistic modeling of oceanic wakes. For instance, the velocity and the geopotential deviation of the upstream flow are generally nonuniform along the spanwise direction, and the bottom slope topography cannot be neglected in coastal regions. Moreover, the vertical stratification is often more complex than a simple two-layer configuration. All these effects could influence the cyclone-anticyclone asymmetry in the vortex street observed in the laboratory experiment.

\section{ACKNOWLEDGMENTS}

The authors acknowlege Claire Donnadieu and Alexandre Pereira for their participation to the laboratory work. The authors also wish to thank Jean-Marc Chomaz and Xavier Carton for fruitful discussions, as well as Jim McWilliams for all his valuable remarks and comments on this manuscript.

\section{APPENDIX: LIST OF EXPERIMENTS}

\begin{tabular}{cccccccc} 
Expt. & $R_{d}(\mathrm{~cm})$ & $\mathrm{Bu}$ & $\mathrm{Ro}$ & $\mathrm{Re}$ & $\mathrm{Fr}$ & $\lambda$ & $\mathrm{St}$ \\
\hline 1 & 23.8 & 46.2 & 0.13 & 196 & 0.02 & 0.003 & 0.27 \\
2 & 11.9 & 11.5 & 0.06 & 196 & 0.02 & 0.006 & 0.27 \\
3 & 9.63 & 7.6 & 0.13 & 400 & 0.05 & 0.02 & 0.23 \\
4 & 4.91 & 1.97 & 0.06 & 196 & 0.04 & 0.03 & 0.24 \\
5 & 4.90 & 1.96 & 0.35 & 980 & 0.25 & 0.18 & 0.31 \\
6 & 3.60 & 1.06 & 0.36 & 1015 & 0.35 & 0.34 & 0.37 \\
7 & 3.50 & 1.00 & 0.35 & 1078 & 0.35 & 0.35 & 0.39 \\
8 & 3.48 & 1.00 & 0.06 & 196 & 0.06 & 0.06 & 0.28 \\
9 & 3.48 & 0.99 & 0.25 & 771 & 0.25 & 0.25 & 0.32 \\
10 & 2.89 & 0.68 & 0.06 & 196 & 0.07 & 0.09 & 0.30
\end{tabular}




\begin{tabular}{cccccccc} 
Expt. & $R_{d}(\mathrm{~cm})$ & $\mathrm{Bu}$ & $\mathrm{Ro}$ & $\mathrm{Re}$ & $\mathrm{Fr}$ & $\lambda$ & $\mathrm{St}$ \\
\hline 11 & 2.76 & 0.62 & 0.18 & 763 & 0.23 & 0.29 & 0.31 \\
12 & 2.44 & 0.49 & 0.10 & 400 & 0.14 & 0.20 & 0.28 \\
13 & 2.40 & 0.47 & 0.33 & 938 & 0.48 & 0.70 & 0.38 \\
14 & 2.40 & 0.47 & 0.34 & 966 & 0.50 & 0.72 & 0.45 \\
15 & 2.06 & 0.35 & 0.06 & 196 & 0.10 & 0.18 & 0.33 \\
16 & 1.92 & 0.30 & 0.19 & 785 & 0.35 & 0.63 & 0.41 \\
17 & 1.70 & 0.24 & 0.24 & 1000 & 0.49 & 1.00 & \\
18 & 1.67 & 0.23 & 0.11 & 400 & 0.23 & 0.48 & 0.32 \\
19 & 1.60 & 0.21 & 0.19 & 771 & 0.42 & 0.9 & 0.46 \\
20 & 1.43 & 0.17 & 0.18 & 763 & 0.44 & 1.06 & 0.48 \\
21 & 1.31 & 0.14 & 0.05 & 196 & 0.13 & 0.34 & 0.32 \\
22 & 1.17 & 0.11 & 0.08 & 400 & 0.24 & 0.71 & 0.41 \\
23 & 1.17 & 0.11 & 0.19 & 798 & 0.57 & 1.71 & 0.56
\end{tabular}

${ }^{1}$ B. A. Smith, L. A. Soderblom, and T. V. Johnson, "Jupiter system through the eyes of Voyager-1," Science 204, 951 (1979).

${ }^{2}$ G. P. Williams and T. Yamagata, "Geostrophic regimes, intermediate solitary vortices and Jovian eddies," J. Atmos. Sci. 41, 453 (1984).

${ }^{3}$ B. A. Smith, L. A. Soderblom, and R. Batson, "A new look at the Saturn system: The Voyager-2 images," Science 215, 504 (1982).

${ }^{4}$ B. A. Smith, L. A. Soderblom, and D. Banfield, "Voyager-2 at Neptune: Imaging science results," Science 246, 1422 (1989).

${ }^{5}$ J. C. McWilliams, "Submesoscale, coherent vortices in the ocean," Rev. Geophys. 23, 165 (1985).

${ }^{6}$ X. Carton, "Hydrodynamical modeling of oceanic vortices," Surv. Geophys. 22, 179 (2001).

${ }^{7}$ T. Matsuura and T. Yamagata, "On the evolution of nonlinear planetary eddies larger than the radius of deformation," J. Phys. Oceanogr. 12, 440 (1982).

${ }^{8}$ V. I. Petviashvili, "Red spot of Jupiter and the drift soliton in a plasma," JETP Lett. 32, 632 (1980).

${ }^{9}$ S. D. Meyers, J. Sommeria, and H. L. Swinney, "Laboratory study of the dynamics of jovian-type vortices," Physica D 37, 515 (1989).

${ }^{10}$ J. Nycander and G. G. Sutyrin, "Stationary translating anticyclones on the beta-plane," Dyn. Atmos. Oceans 16, 473 (1992).

${ }^{11}$ A. Stegner and V. Zeitlin, "Asymptotic expansions and monopolar solitary rossby vortices in barotropic and two-layer models," Geophys. Astrophys. Fluid Dyn. 83, 159 (1996).

${ }^{12}$ L. M. Polvani, J. C. McWilliams, M. A. Spall, and R. Ford, "The coherent structures of shallow-water turbulence: Deformation-radius effects, cyclone/anticyclone asymmetry and gravity-wave generation," Chaos $\mathbf{4}$, 177 (1994).

${ }^{13}$ M. Arai and T. Yamagata, "Asymmetric evolution of eddies in rotating shallow water," Chaos 4, 163 (1994).

${ }^{14}$ M. Farge and R. Sadourny, "Wave-vortex dynamics in rotating shallow water," J. Fluid Mech. 206, 433 (1989).

${ }^{15}$ A. Stegner and D. G. Dritschel, "A numerical investigation of the stability of isolated shallow water vortices," J. Phys. Oceanogr. 30, 2562 (2000).

${ }^{16}$ F. J. Poulin and G. R. Flierl, "The nonlinear evolution of barotropically unstable jets," J. Phys. Oceanogr. 33, 2173 )2003).

${ }^{17}$ L. P. Graves, J. C. McWilliams, and M. T. Montgomery, "Vortex evolution due to straining: A mechanism for dominance of strong, interior anticyclones," Geophys. Astrophys. Fluid Dyn. (submitted).

${ }^{18}$ P. F. Linden, B. M. Boubnov, and S. B. Dalziel, "Source-sink turbulence in a rotating stratified fluid," J. Fluid Mech. 298, 81 (1995).

${ }^{19}$ S. Yanase, C. Flores, O. Metais, and J. Riley, "Rotating free-shear flows. I. Linear stability analysis," Phys. Fluids A 5, 2725 (1993).

${ }^{20} \mathrm{~T}$. Matsuura, "The evolution of frontal-geostrophic vortices in a two-layer ocean," J. Phys. Oceanogr. 25, 2298 (1995).

${ }^{21}$ J. M. Baey and X. Carton, "Vortex multipoles in two-layer rotating shallow-water flows," J. Fluid Mech. 460, 151 (2002).

${ }^{22}$ J. Pedlosky, Geophysical Fluid Dynamics (Springer, New York, 1987).

${ }^{23}$ B. Cushman-Roisin, G. G. Sutyrin, and B. Tang, "Two-layer geostrophic dynamics. Part I: Governing equations," J. Phys. Oceanogr. 22, 117 (1992).

${ }^{24}$ C. Y. Wen and C. Y. Lin, "Two dimensionnal vortex shedding of a circular cylinder," Phys. Fluids 13, 557 (2001).

${ }^{25}$ G. EM. Karniadakis and G. S. Triantafyllou, "Frequency selection and asymptotic states in laminar wakes," J. Fluid Mech. 199, 441 (1988)

${ }^{26}$ C. H. K. Williamson, "Vortex dynamics in the cylinder wake," Annu. Rev. Fluid Mech. 28, 477 (1996).

${ }^{27}$ D. Barkley and R. D. Henderson, "Three-dimensional Floquet stability analysis of the wake of a circular cylinder," J. Fluid Mech. 322, 215 (1996).

${ }^{28}$ G. Chabert D'Hieres, P. A. Davies, and H. Didelle, "A laboratory study of the lift forces on a moving solid obstacle in a rotating fluid," Dyn. Atmos. Oceans 13, 47 (1989).

${ }^{29}$ J. A. Johnson, "Stability of shearing motion in rotating fluid," J. Fluid Mech. 17, 337 (1963).

${ }^{30}$ S. Yanase, C. Flores, O. Metais, and J. Riley, "Rotating free-shear flows. I. Linear stability analysis," Phys. Fluids A 5, 2725 (1993).

${ }^{31}$ A. C. Kuo and L. M. Polvani, "Nonlinear geostrophic adjustment, cyclone/ anticyclone asymmetry, and potential vorticity rearrangement," Phys. Fluids 12, 1087 (2000).

${ }^{32}$ G. Chabert D'Hieres, P. A. Davies, and H. Didelle, "Experimental studies of lift and drag forces upon cylindrical obstacles in homogeneous rapidly rotating fluids," Dyn. Atmos. Oceans 15, 87 (1990).

${ }^{33}$ D. L. Boyer and M. L. Kmetz, "Vortex shedding in rotating flows," Geophys. Astrophys. Fluid Dyn. 26, 51 (1983).

${ }^{34}$ D. L. Boyer, M. L. Kmetz, and L. Smathers, "Rotating open channel flow past right circular cylinders," Geophys. Astrophys. Fluid Dyn. 30, 271 (1984).

${ }^{35}$ A. Stegner, T. Pichon, and M. Beunier, "Elliptical-inertial instability of rotating Karman streets," Phys. Fluids 17, 066602 (2005).

${ }^{36}$ B. Cushman-Roisin, "Frontal geostrophic dynamics," J. Phys. Oceanogr. 16, 132 (1986).

${ }^{37}$ D. D. Holm, J. E. Mardsen, T. Ratieu, and A. Weinstein, "Nonlinear stability of fluid and plasma equilibria," Phys. Rep. 123, 1 (1985).

${ }^{38} \mathrm{M}$. Ben Jelloul and V. Zeitlin, "Remarks on stability of the rotating shallow-water vortices in the frontal dynamics regime," Nuovo Cimento Soc. Ital. Fis., C 22, 931 (1999). 